\section{Early recognition and manage- ment of septic shock in children}

\author{
Paolo Biban, Marcella Gaffuri, \\ Stefania Spaggiari, Federico Zaglia, \\ Alessandra Serra, Pierantonio Santuz \\ Department of Pediatrics, Pediatric and \\ Neonatal intensive Care Unit, Azienda \\ Ospedaliera Universitaria Integrata, \\ Verona, Italy
}

\section{Abstract}

Septic shock remains a major cause of morbidity and mortality among children, mainly due to acute hemodynamic compromise and multiple organ failures. In the last decade, international guidelines for the management of septic shock, as well as clinical practice parameters for hemodynamic support of pediatric patients, have been published. Early recognition and aggressive therapy of septic shock, by means of abundant fluid resuscitation, use of catecholamines and other adjuvant drugs, are widely considered of pivotal importance to improve the short and long-term outcome of these patients. The aim of this paper is to summarize the modern approach to septic shock in children, particularly in its very initial phase, when pediatric healthcare providers may be required to intervene in the pre-intensive care unit setting or just on admission in the pediatric intensive care unit.

\section{Introduction}

Severe sepsis and septic shock constitute a relevant cause of morbidity and mortality in critically ill children.1-3 Actually, most deaths from sepsis occur globally in locations without intensive care units, and many of them could be prevented using relatively simple measures as recommended by the World Health Organization-Integrated Management of Childhood Illnesses guidelines. ${ }^{4}$ On this regard, The World Federation of Pediatric Intensive Care and Critical Care Societies (WFPICCS) has recently launched an important quality improvement program (The Global Pediatric Sepsis Initiative at http://www. wfpiccs.org/sepsis and http//www. pediatricsepsis.org), with the goal of ameliorating the outcome of septic children worldwide, regardless the amount of available resources. ${ }^{5}$

Nonetheless, septic shock still represent a clinical challenge even in developed countries, being a leading causes of admissions to the paediatric emergency department and the pediatric intensive care unit.

In the last decades, several consensus conferences about the criteria for defining sepsis and related conditions have been held. ${ }^{6}$ However, definitions of sepsis, severe sepsis, septic shock and multiple organ dysfunction/failure syndromes in children are slightly different when compared to those used for adults. In particular, septic shock in children is defined as presence of sepsis plus signs of cardiovascular organ dysfunction, not necessarily including hypotension, whilst in adult septic shock hypotension must be pres-

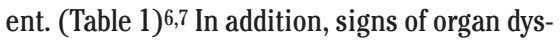
function depend on age-specific heart rate, respiratory rate, and white blood cell count cutoffs, which characteristically change in different paediatric age groups.

Guidelines for the management of severe sepsis and septic shock, sponsored by the International Surviving Sepsis Campaign, and clinical practice parameters released by the American College of Critical Care Medicine (ACCM) for hemodynamic support of paediatric and neonatal septic shock have been published and recently updated. $8-11$

The aim of this paper is to summarize the modern approach to septic shock in children in its very initial phase, emphasising the pivotal role of early recognition and prompt management of this life-threatening condition, in PICU but also in the pre-ICU setting.

\section{Pathophysiology of septic shock}

Shock is a complex clinical syndrome caused by an acute failure of circulatory function, with inadequate tissue and organ perfusion, where delivery of oxygen and substrates to body tissues, as well as removal of metabolic waste products are inadequate. This results in cellular dysfunction, which may eventually lead to cell death. Shock is the common endpoint of many pathophysiological pathways. There are three major categories of shock: hypovolemic, cardiogenic and distributive, with a degree of overlap between these. Septic shock usually fall into the categories of distributive and hypovolemic shock. In the distributive shock is prevalent a circulatory maldistribution associated with peripheral vasodilatation, arterial and capillary shunting, whereas in the hypovolemic shock there is inadequate circulating blood volume, despite peripheral vasoconstriction.

Hypotension frequently constitutes one of the prominent features of shock, but could be a late sign in pediatric patients. $7,10,11$

The most common causes of septic shock are of bacterial origin, a classic example being meningococcal disease. However, any organ-
Correspondence: Paolo Biban, Pediatric and Neonatal intensive Care Unit, Department of Pediatrics, Azienda 0spedaliera Universitaria Integrata, piazzale Stefani 1, 37126, Verona, Italy. Tel: +39.045 .8122365 - Fax: +39.045 .8123373$

E-mail: paolo.biban@ospedaleuniverona.it

Key words: septic shock, children.

Received for publication: 3 November 2011. Accepted for publication: 23 February 2012.

This work is licensed under a Creative Commons Attribution NonCommercial 3.0 License (CC BYNC 3.0).

(C) Copyright P. Biban et al., 2012

Licensee PAGEPress, Italy

Pediatric Reports 2012; 4:e13

doi:10.4081/pr.2012.e13

ism can precipitate severe sepsis and septic shock, including bacteria, viruses, mycobacteria and fungi, especially in the immunocompromised host.

\section{Early recognition of paediatric septic shock}

In the management of septic shock, early diagnosis and prompt treatment have the greatest impact on clinical course and patient outcome. Indeed, early recognition of septic shock and institution of antibiotic therapy can reduce mortality in children. ${ }^{12,13}$ Furthermore, early institution of resuscitative measures, particularly by using aggressive fluid replacement strategies, may prevent the child from entering uncompensated or irreversible shock, thereby reducing morbidity and mortality.

However, diagnosis of sepsis can be particularly difficult in children, in whom a specific signs of sepsis, e.g. tachycardia, tachypnoea and fever, need a special interpretation due to the variable range of normality depending on the patient age. Furthermore, previously healthy children with intact cardiovascular homeostatic mechanisms can compensate extremely well during hypoperfusion states for relatively long periods. Thus, shock in children should be suspected by clinical and laboratory signs, including altered mental status, tachypnoea and tachycardia, hypothermia or hyperthermia, changes in peripheral perfusion, together with reduction of urine output, metabolic acidosis and increased blood lactate. Importantly, hypotension is not necessary for the clinical diagnosis of septic shock, even though its presence in any child with clinical suspicion of infection is confirmatory. 7,11

In the emergency department setting, chil- 
dren with severe infections and changes in peripheral perfusion are frequently described as in warm shock or cold shock status, on the basis of first clinical examination (Table 2). ${ }^{14}$

Early in the course of the disease process, common observations usually include abnormal temperature regulation, flushed warm skin, a widened pulse pressure (warm shock), tachycardia, tachypnoea, whereas mean arterial pressure is frequently maintained. Clinical signs of late septic shock include hypotension, tachycardia with narrow pulse pressure, cold extremities (cold shock), rapid shallow breathing, oliguria, altered level of consciousness, and cyanosis due to pulmonary ventilation perfusion mismatch or underlying pulmonary diseases. At present, international consensus recommends early recognition of pediatric septic shock using simply clinical examination, not biochemical tests, event though some experts suggest to consider lactate levels as well.11

\section{Early management of paedi- atric septic shock: the golden hour}

Shock can rapidly evolve through different phases, usually from a compensated to an uncompensated status, which may ultimately become irreversible. Aggressive treatment should be initiated in all cases where shock is suspected. In fact, the mortality and outcome of septic shock is likely influenced by the speed and appropriateness of therapy administered in the initial hours after the syndrome develops. ${ }^{12,15,16}$ The goal is to reverse shock and prevent multiple organ dysfunction. To achieve this, the initial management of septic shock (first hour) should focus on some major therapeutic end points of resuscitation, which include normalization of heart rate and blood pressure (adjusted for age), capillary refill of less than 2 seconds, normal pulses with no differential between peripheral and central pulses, warm extremities, normal mental status, urine output greater than $1 \mathrm{~mL} / \mathrm{kg} / \mathrm{hr}$. In addition, normal glucose and ionized calcium concentrations should be achieved and maintained. ${ }^{11}$

Once shock is suspected, appropriate supportive measures must be instituted as soon as possible, ideally following the ACCM guidelines. ${ }^{11}$ These include securing airway patency, providing supplemental oxygen and positivepressure ventilation, achieving vascular access, infusing as many fluids as needed, starting empiric antibiotic therapy, and providing support with inotropes and vasopressors, as clinically indicated. Importantly, empiric antibiotic treatment should not be delayed while waiting blood cultures to be sampled.

Table 1. Cardiovascular dysfunction criteria (modified from Goldstein et al.7).

\begin{tabular}{|c|c|c|}
\hline $\begin{array}{l}\text { Decrease in blood pressure } \\
\text { (hypotension) }<5 \text { th percentile } \\
\text { for age or systolic BP }<2 \mathrm{SD} \\
\text { below normal for age }\end{array}$ & $\begin{array}{l}\text { Need for vasoactive drug } \\
\text { to maintain blood pressure } \\
\text { in normal range (dopamine } \\
>5 \mathrm{mcg} / \mathrm{kg} / \mathrm{min} \text { or dobutamine, } \\
\text { adrenaline, or noradrenaline } \\
\text { at any dose) }\end{array}$ & $\begin{array}{l}\text { Two of the following: } \\
\text { 1) unexplained metabolic } \\
\text { acidosis: base deficit }>5.0 \mathrm{mEq} / \mathrm{L} \\
\text { 2) Increased arterial lactate }>2 \\
\text { times upper limit of normal } \\
\text { 3) Oliguria: urine output }<0.5 \mathrm{~mL} / \mathrm{kg} / \mathrm{hr} \\
\text { 4) Prolonged capillary refill: }>5 \mathrm{secs} \\
\text { 5) Core to peripheral temperature gap }>3^{\circ} \mathrm{C}\end{array}$ \\
\hline
\end{tabular}

Two levels of care can be then summarized: first, early fluid load, mostly for outpatients with community acquired infections; second, advanced technology for children with fluid/dopamine refractory illness, scarcely responding to the initial stabilization attempts. These interventions can be located at the onset and at the end of the golden hour, according to international guidelines, where time intervals for intensivists have been clearly indicated.11 Indeed, the early interpretation of hemodynamic pattern of septic shock can guide further therapeutic interventions and vasoactive agents introduction. Thus, early referral to highly specialised centres, capable to provide more sophisticated monitoring and treatment, is strongly recommended.

\section{$A B C$ s: airway and breathing}

Airway, breathing and circulation remain the fundamental principles of resuscitation also in patients with septic shock. Priority is given to the airway, which is immediately assessed and secured, if necessary. High flow oxygen via facemask or nasal canulae should be given, even in the absence of respiratory distress or hypoxemia.

Aggressive airway management and ventilation should be considered in any patient not responding to fluid resuscitation and peripherally administered inotropes. Indeed, early intubation and mechanical ventilation may support cardiac output (CO) by reducing the work of breathing, reduce oxygen consumption by sedation, facilitate procedures for establishing invasive hemodynamic monitoring, provide airway protection. ${ }^{11}$

\section{Vascular access}

Rapid attainment of a vascular access is of critical importance, possibly through the placement of two large bore peripheral venous catheters. If these catheters cannot be obtained easily, the intraosseous route or a central venous catheter should be considered, depending upon the available expertise.

If at all possible, blood samples should be taken for haematological parameters, urea and electrolytes, blood glucose, culture and crossmatch. At this point empirical antibiotic therapy should be started intravenously.
Table 2. Clinical characteristics of cold and warm shock (modified from Saladino et al. ${ }^{14}$ ).

$$
\text { Cold shock Warm shock }
$$

Capillary > 2 seconds Flash capillary refill refill

\begin{tabular}{lll}
$\begin{array}{l}\text { Peripheral } \\
\text { pulses }\end{array}$ & Diminished & Bounding \\
\hline $\begin{array}{l}\text { Mottling } \\
\text { of skin }\end{array}$ & Present & Absent \\
\hline
\end{tabular}

of skin

\section{Fluid resuscitation}

Rapid fluid boluses of $20 \mathrm{~mL} / \mathrm{kg}$ should be administered (possibly over five minutes), observing for the development of lung rales or hepatomegaly. Up to $60 \mathrm{~mL} / \mathrm{kg}$ may be necessary in the first hour; however, in some patients aliquots exceeding $150-200 \mathrm{ml} / \mathrm{kg}$ may be required in the first hour. Fluid should be pushed manually or by a pressure bag, with a goal of attaining normal perfusion and blood pressure.11 Use of both crystalloid or colloid solutions is generally considered appropriate. In special cases, e.g. when the source of hypovolemia is hemorrhage, transfusion with packed red blood cells could be also considered, particularly with haemoglobin values below 10 $\mathrm{ng} / \mathrm{dL}$.

However, there is little evidence about the best type of resuscitation fluid, the appropriate timing, volume, and rate of fluid administration. ${ }^{17}$ In particular, no clear advantages have been demonstrated by using cristalloids rather than colloids in septic shock patients. ${ }^{18}$ Interestingly, recent findings challenge the importance of bolus resuscitation as a lifesaving intervention in resource-limited settings for children with shock who do not have hypotension, raising questions regarding fluid-resuscitation guidelines in other settings as well. ${ }^{19}$ Further research is clearly needed to clarify such a controversial issue.

\section{Inotropic support}

Children with septic shock uniformly require some vasoactive support during and after fluid resuscitation. Importantly, in patients not responsive to fluid resuscitation, inotropic support should be started via a sec- 
ond peripheral access, until a safer central venous access is obtained.

Dopamine can be used and titrated as the first-line agent, up to $10 \mathrm{mcg} / \mathrm{kg} / \mathrm{min}$. However, if the patient is not rapidly responsive to therapy, then adrenaline or noradrenaline should be infused to restore normal blood pressure and perfusion. ${ }^{9,11}$ In general, adrenaline ranging from 0.05 to $0.3 \mathrm{mcg} / \mathrm{kg} / \mathrm{min}$ should be used in patients with normal or low blood pressure and cold shock, whilst noradrenaline should be titrated in patients with low blood pressure and warm shock.

\section{Corticosteroids}

Some patients with septic shock may suffer of severe adrenal insufficiency, which could partially explain a scarce response to fluid and catecholamine therapy. Risk factors for such condition include purpura fulminans, prior corticosteroid use and pituitary or adrenal abnormalities. In patients with fluid refractory, catecholamine resistant shock, intermittent or continuous infusions of hydrocortisone, ranging from $1 \mathrm{up}$ to $50 \mathrm{mg} / \mathrm{kg} /$ day is recommended within 60 min of diagnosis of shock..$^{9,11}$

\section{Other therapies for acute manage- ment of septic shock}

Beyond the first hour of treatment, several adjuvant therapies, including vasopressin, terlipressin, levosimendan, milrinone, immunoglobulins, and protein C concentrate, have been attempted in pediatric septic shock, even though evidence to support their use are still lacking. $9,11,20-24$

Finally, children with septic shock scarcely responding to initial aggressive treatments must be suspected to have unrecognized morbidities, including pericardial effusion, pneumothorax, ongoing blood loss, hypoadrenalism, hypothyroidism, inborn errors of metabolism, or congenital heart disease. When these causes have been excluded, extreme therapeutic options such as extracorporeal membrane oxygenation (ECM0) should be considered. ${ }^{9-11}$

\section{Advanced non invasive hemodynamic monitoring}

The invasive monitoring of cardiac output is not common practice in the pediatric emergency department. Interestingly, newer, noninvasive, ultrasound techniques offer the opportunity to monitor the hemodynamic patterns of children with septic shock, at a much earlier stage than that usually accomplished in the intensive care unit.

In a recent study, the hemodynamic patterns of children with suspected fluid-resistant septic shock was investigated by means of a noninvasive cardiac output device. Patients who presented with central venous catheter-related sepsis predominantly demonstrated a pattern of elevated cardiac index with low systemic vascular resistance (warm shock), whereas those who presented with community acquired sepsis predominantly exhibited a low or normal cardiac index, with normal or high systemic vascular resistance (cold shock). ${ }^{25}$

Theoretically, such devices may provide a more specific assessment of the hemodynamic status of these patients, even in the emergency department or during transportation, helping the clinician to individualize the therapeutic management.

\section{Transfer of patients with septic shock to a referral centre}

Even when initial stabilisation has been correctly achieved, the early referral to a highlevel PICU may be life-saving for a child with evolving septic shock. Indeed, more advanced monitoring and treatment are best offered in an experienced PICU setting. Areas lacking specialised paediatric ICUs should arrange for a transfer system towards a tertiary PICU, ideally by means of a specialised transport team.

\section{Conclusions}

Septic shock in children still constitutes a clinical challenge for healthcare providers, both in the emergency department and the intensive care unit. Early diagnosis, allowing rapid therapeutic intervention, is essential in improving the outcome of these patients. Guidelines for the management of septic shock have been published and are regularly updated by highly recognized international bodies. Current treatment includes early fluid resuscitation, tailored use of inotropes and vasopressors, and use of adjuvant treatments, such as hydrocortisone. Novel promising therapies are on the horizon, but hitherto they remain largely unproven in terms of efficacy and safety.

Even though remarkable advances have been made in the management of paediatric septic shock, further research is still needed in order to improve the short and long-term outcome of these high-risk patients.

\section{References}

1. Watson RS, Carcillo JA, Linde-Zwirble WT, et al. The epidemiology of severe sepsis in the United States. Am J Respir Crit Care Med 2003;167:695-701.

2. Angus DC, Linde Zwirble WT, Liddicker J, et al. Epidemiology of severe sepsis in the U.S.: Analysis of incidence, outcome, and associated costs of care. Crit Care Med 2001;29:1303-10.

3. Odetola F0, Gebremariam A, Freed GL. Patient and hospital correlates of clinical outcomes and resource utilization in severe pediatric sepsis. Pediatrics. 2007;119:487-94.

4. Integrated Management of Childhood Illnesses: WHO/UNICEF Initiative Integrated Management of Childhood Illness. Available from: http://whqlib doc.who.int/publications/2005/9241546 441.pdf. Accessed: November 3, 2011

5. Kissoon N, Carcillo J, Espinosa V, et al. The Global Sepsis Initiative Vanguard Center Contributors. World Federation of Pediatric Intensive Care and Critical Care Societies. Pediatr Crit Care Med 2011;12: 494-503.

6. Levy MM, Fink MP, Marshall JC, et al. 2001 SCCM/ESICM/ACCP/ATS/SIS International Sepsis Definitions Conference. Crit Care Med 2003;31:1250-6.

7. Goldstein B, Giroir B, Randolph A. International pediatric sepsis consensus conference: Definitions for sepsis and organ dysfunction in pediatrics. Pediatr Crit Care Med 2005; 6:2-8.

8. Dellinger RP, Carlet JM, Masur H, et al. Surviving Sepsis Campaign guidelines for management of severe sepsis and septic shock. Intensive Care Med 2004;30:536-55.

9. Dellinger RP, Levy MM, Carlet JM, et al. Surviving Sepsis Campaign: International guidelines for management of severe sepsis and septic shock: 2008. Crit Care Med 2008;36:296-327.

10. Aneja RK, Carcillo J. Differences between adult and pediatric septic shock. Minerva Anestesiol 2011;77:986-92.

11. Brierley J, Carcillo JA, Choong K, et al. Clinical practice parameters for hemodynamic support of pediatric and neonatal septic shock: 2007 update from the American College of Critical Care Medicine. Crit Care Med 2009;37:666-88.

12. Han YY, Carcillo JA, Dragotta MA, et al. Early reversal of pediatric-neonatal septic shock by community physicians is associated with improved outcome. Pediatrics 2003;112:793-9.

13. Inwald DP, Tasker RC, Peters MJ, Nadel S. Emergency management of children with severe sepsis in the United Kingdom: the results of the Paediatric Intensive Care Society sepsis audit. Arch Dis Child 2009; 94:348-53.

14. Saladino RA. Management of septic shock in the pediatric emergency department in 2004. Clin Ped Emerg Med 2004;5:20-27.

15. Melendez E, Bachur R. Advances in the emergency management of pediatric sepsis. Curr Opin Pediatr 2006;18: 245-53.

16. de Oliveira CF, de Oliveira DS, Gottschald AF, et al: ACCM/PALS haemodynamic support guidelines for paediatric septic shock: an outcomes comparison with and without monitoring central venous oxygen satura- 
tion. Intensive Care Med 2008;34:1065-75.

17. Myburgh JA. Fluid resuscitation in acute illness - Time to reappraise the basics. $\mathrm{N}$ Engl J Med 2011;364: 2543-4.

18. Akech S, Ledermann H, Maitland K. Choice of fluids for resuscitation in children with severe infection and shock: systematic review. BMJ 2010;341:c4416.

19. Maitland K, Kiguli S, Opoka RO, et al. Mortality after fluid bolus in African children with severe infection. $\mathrm{N}$ Engl J Med 2011;364: 2483-95.

20. Choong K, Bohn D, Fraser DD, et al.
Vasopressin in pediatric vasodilatory shock: a multicenter randomized controlled trial. Am J Respir Crit Care Med 2009;180:632-9.

21. Yildizdas D, Yapicioglu H, Celik U, et al. Terlipressin as a rescue therapy for catecholamine-resistant septic shock in children. Intensive Care Med 2008;34:511-7.

22. Biban P, Gaffuri M. Role of vasopressin and terlipressin in neonates and children with refractory septic shock. Curr Drug Metab 2012 (in press)

23. Barton P, Garcia J, Kouatli A, et al.
Hemodynamic effects of i.v. milrinone lactate in pediatric patients with septic shock. A prospective, double-blinded, randomized, placebo-controlled, interventional study. Chest 1996;109:1302-12.

24. Namachivayam P, Crossland DS, Butt WW, et al: Early experience with levosimendan in children with ventricular dysfunction. Pediatr Crit Care Med 2006;7:445-8.

25. Brierley J, Peters MJ. Distinct hemodynamic patterns of septic shock at presentation to pediatric intensive care. Pediatrics 2008;122;752-9. 